\title{
Trends in redo mitral procedure for treating mitral bioprostheses failure: a single center's experience
}

\author{
Jian Liu ${ }^{1 \#}$, Peijian Wei ${ }^{1,2 \#}$, Yanjun Liu ${ }^{1 \#}$, Jiexu Ma ${ }^{1,2}$, Hongxiang $\mathrm{Wu}^{1}$, Tong $\operatorname{Tan}^{1,2}$, Zhao Chen ${ }^{1}$, Jimei Chen ${ }^{1}$, \\ Huanlei Huang ${ }^{1}$, Huiming Guo ${ }^{1}$ \\ ${ }^{1}$ Department of Cardiovascular Surgery, Guangdong Cardiovascular Institute, Guangdong Provincial People's Hospital (Guangdong Academy of \\ Medical Sciences), Guangzhou, China; ${ }^{2}$ Shantou University Medical College, Shantou, China \\ Contributions: (I) Conception and design: J Liu, P Wei, Y Liu; (II) Administrative support: J Chen, H Huang, H Guo; (III) Provision of study materials \\ or patients: J Liu, H Huang, H Guo; (IV) Collection and assembly of data: J Ma, H Wu, T Tan, Z Chen; (V) Data analysis and interpretation: J Liu, \\ P Wei, Y Liu; (VI) Manuscript writing: All authors; (VII) Final approval of manuscript: All authors. \\ \#These authors contributed equally to this work. \\ Correspondence to: Huiming Guo; Huanlei Huang. Department of Cardiovascular Surgery, Guangdong Cardiovascular Institute, Guangdong \\ Provincial People's Hospital (Guangdong Academy of Medical Sciences), Guangzhou 510080, China. Email: guohuiming@gdph.org.cn; \\ huanghuanlei@gdph.org.cn.
}

Background: Transcatheter mitral valve-in-valve implantation (TM-VIV) has emerged as a viable and attractive alternative to surgical mitral valve replacement (SMVR). This study aimed to review a singlecenter experience with redo mitral procedure for mitral bioprostheses failure over an 8-year period. In addition, it compared procedural safety and early outcomes of various approaches.

Methods: Between January 2013 and January 2021, 79 consecutive patients who underwent redo procedure for mitral bioprostheses failure in our institution were retrospectively reviewed. SMVR and transapical TMVIV were performed in 54 and 25 patients, respectively. In the SMVR group, 12 patients underwent totally thoracoscopic redo mitral valve replacement (MVR).

Results: The annual volume of procedures grew continuously during the study period, with the use of totally thoracoscopic redo MVR increasing from $0 \%$ in 2012 to $20 \%$ in 2019 . In 2020 , $84.2 \%$ of total procedures were performed via the transcatheter approach. Patients in the TM-VIV group were significantly older and had higher scores on the European System for Cardiac Operative Risk Evaluation II (EuroScore II) and the Society of Thoracic Surgeons Predicted Risk of Mortality (STS PROM) $(\mathrm{P}<0.01)$. The in-hospital mortality for the SMVR group and TM-VIV group was 3.7\% (2 patients) and 0, respectively. Compared to the SMVR group, TM-VIV was associated with shorter ventilation time, intensive care unit stay, and postoperative in-hospital stay, and there was less need for blood transfusion. In the subgroup analysis, no significant difference was detected among most perioperative outcomes between the totally thoracoscopy approach group and the TM-VIV group.

Conclusions: There is an increasing number of patients demanding surgical treatments for mitral bioprostheses failure. TM-VIV is playing a significant role due to its scope of application and excellent outcomes.

Keywords: Redo surgical mitral valve replacement (SMVR); transcatheter mitral valve-in-valve implantation (TM-VIV); transapical; mitral bioprostheses failure

Submitted Jun 02, 2021. Accepted for publication Jul 07, 2021.

doi: $10.21037 / \mathrm{atm}-21-3118$

View this article at: https://dx.doi.org/10.21037/atm-21-3118 


\section{Introduction}

The evolution of mitral valve surgery has been driven by the demand for less invasive therapy that does not compromise the quality of the procedure. Since the 1960s, mitral valve surgery has progressed from conventional median sternotomy to mini-thoracotomy, video-assisted thoracoscopic, totally thoracoscopic, robot-assisted, and transcatheter approaches. In Germany, minimally invasive mitral valve surgery increased from $13.1 \%$ in 2004 (1) to $45.5 \%$ in 2019 (2). Although a minimally invasive approach has been the most commonly adopted option since 2017 (3), transcatheter mitral valve surgery is emerging as an attractive and competitive option, with its use increasing from $0.5 \%$ in 2011 (4) to $15.1 \%$ in 2019 (2). The past two decades have witnessed a rise in the use of bioprosthetic valves (5), but this procedure inevitably leads to reoperation due to structural deterioration. Data on reoperation trends for failed mitral bioprostheses in mainland China are limited. In this study, we aimed to review our experience with redo mitral procedure for mitral bioprostheses failure over an 8-year period. We present the following article in accordance with the STROBE reporting checklist (available at https://dx.doi. org/10.21037/atm-21-3118).

\section{Methods}

This retrospective, observational, cohort study collected data from consecutive patients who underwent redo mitral valve procedures for failed bioprostheses at Guangdong Provincial People's Hospital between January 2013 and January 2021. We excluded patients who required concomitant procedures for coronary artery disease or aortic disease or had active endocarditis. Among the enrolled patients, 54 patients underwent surgical mitral valve replacement (SMVR) and 25 underwent transapical transcatheter mitral valve-in-valve implantation (TM-VIV). SMVR was performed via median sternotomy in 42 patients and via the totally thoracoscopic approach in 12 patients. The study was approved by the Ethics Committee of Guangdong Provincial People's Hospital (No. KY-Q-2021-088-01) and individual consent was waived. The study was conducted in accordance with the Declaration of Helsinki (as revised in 2013).

\section{Surgical techniques}

\section{Median sternotomy}

Median sternotomy was routinely performed using an oscillating saw. The intrapericardial adhesions were carefully dissected to expose the heart and great vessels. After cardiopulmonary bypass (CPB) was established, standard mitral valve replacement (MVR) was performed conventionally through the interatrial groove under cardioplegia following aortic cross-clamping. The surgical technique has been described in detail elsewhere (6).

\section{Totally thoracoscopic approach}

The operative technique has been described in detail in previous publications (7). A main operating port (3.0-3.5 cm incision) and a thoracoscopic port ( $1.5 \mathrm{~cm}$ incision) were made in the right side of the chest. After systemic heparinization, $\mathrm{CPB}$ was initiated with cannulation through the right femoral artery, right femoral vein, and superior vena cava (through the right jugular vein by an anesthesiologist), under the guidance of transesophageal echocardiography (TEE). Dissection of pleural adhesions was performed if required, and the pericardium was then opened vertically, medial to the phrenic nerve. After the ascending aorta was mobilized and cannulated with a cardioplegia catheter, it was clamped using the Chitwood clamp. The surgical field was flooded with carbon dioxide $\left(\mathrm{CO}_{2}\right)$ to prevent air embolism. The mitral valve was accessed through a left atriotomy in the interatrial groove, and MVR was then performed under cardioplegia. In 10 patients, we failed to successfully dissect the ascending aorta due to dense pericardial adhesion. In these cases, the procedure was performed on the beating heart. After the intracardiac operation, the chest wall was then closed in layers with the application of pericostal sutures to prevent lung herniation.

In the SMVR group, concomitant tricuspid valve surgery was performed if necessary.

\section{Transapical TM-VIV}

The TM-VIV procedure was performed with the J-Valve system (Jiecheng Medical Technologies, Suzhou, China) via a transapical approach in a hybrid operation theater. The sizing of optimal transcatheter heart valve was based on a multimodal evaluation that included direct sizing of the failed prothesis using computed tomography and TEE, and the nominal inner stent diameter of the failed prothesis using the Valve in Valve App (version 2.0, UBQO Limited, London, UK) (8). After general anesthesia, the apex was exposed through a left mini-thoracotomy in the fifth intercostal space and secured by double 3-0 polypropylene purse-string sutures reinforced with Teflon pledgets. A soft J-wire was advanced across the failed bioprosthesis into a pulmonary vein under 


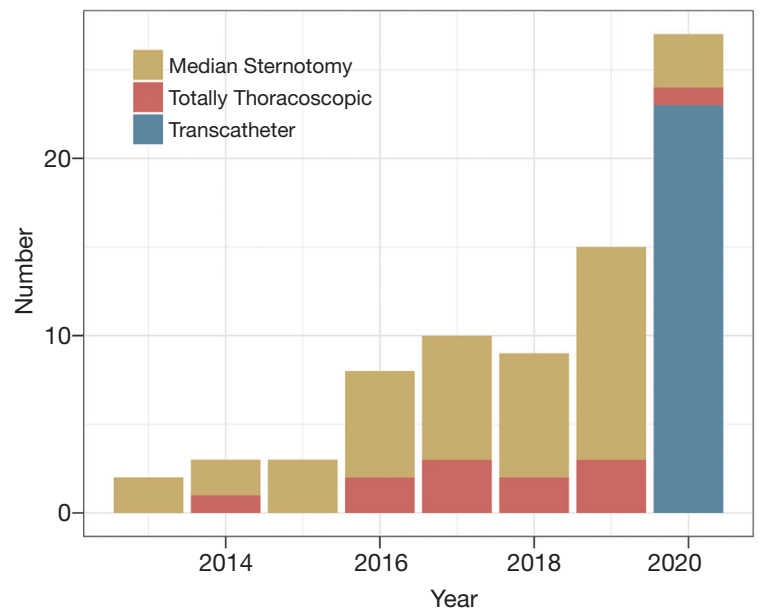

Figure 1 Number of redo MVR for failed mitral bioprosthesis by approach (median sternotomy, totally thoracoscopic, or transcatheter) from 2013 to 2020 . MVR, mitral valve replacement.

fluoroscopy after puncture of the apex. Meanwhile, the $\mathrm{J}$-Valve transcatheter heart valve was reversed and crimped onto the delivery device in ice water. After the soft J-wire was replaced by an extra stiff wire, the J-Valve delivery device was inserted. Subsequently, the J-Valve transcatheter heart valve was placed in the mitral position with guidance of fluoroscopy and TEE. The deployment was performed under rapid ventricular pacing. After no significant paravalvular leakage or transvalvular regurgitation was demonstrated by TEE, the delivery device was removed under a controlled heartbeat. The puncture site was closed, and a drain was placed before closure of chest wall.

\section{Follow-up}

All patients received transthoracic echocardiography assessment before discharge. For the SMVR group, the first follow-up visit was 3 months following hospital discharge. Clinic or telephone follow-ups were then conducted annually. The focus of the follow-up visit was on adverse events, particularly death, reoperation, and recurrent mitral regurgitation. For the TM-VIV group, clinical evaluation and TTE were performed at 30 days, 3 months, 1 year, and yearly thereafter. The follow-up ended on December 30, 2020, for both groups.

\section{Statistical analysis}

Categorical variables are expressed as frequencies and percentages. Continuous variables are presented as mean \pm standard deviation $(M \pm S D)$. When continuous variables were not normally distributed, they are presented as $M$ and interquartile range $\{M,[I Q R]\}$. The Student's $t$-test was used to compare continuous variables. The Mann-Whitney $\mathrm{U}$ test was used to compare continuous and ordinal variables. Associations between categorical variables were evaluated using the chi-squared test or Fisher's exact test as appropriate. A 2-sided $\mathrm{P}$ value of $\leq 0.05$ was considered statistically significant. Statistical analysis was performed using R ( $\mathrm{R}$ x64 version 4.0.2, R Foundation for Statistical Computing, Vienna, Austria).

\section{Results}

Over a period of 8 years, 79 patients with severe symptoms underwent redo mitral valve procedures for failed bioprostheses. The annual volume of procedures increased continuously during the study period. Yearly trend analysis showed a modest increasing use of totally thoracoscopic redo MVR compared to conventional redo MVR from $0 \%$ in 2012 to $20 \%$ in 2019 (Figure 1). Conventional MVR remained the dominant procedure until 2020. Since 2020, TM-VIV has been used in our institution, with $84.2 \%$ of procedures being performed via the transcatheter approach in 2020 .

\section{Patient characteristics}

Baseline characteristics of the study population are shown in Table 1. Compared with the SMVR group, patients who underwent the TM-VIV procedure were more likely to be women $(\mathrm{P}<0.01)$, older $(\mathrm{P}<0.01)$, and with a lower BMI. The TM-VIV group scored significantly higher on the European System for Cardiac Operative Risk Evaluation (EuroScore II) and the Society of Thoracic Surgeons Predicted Risk of Mortality (STS PROM) $(\mathrm{P}<0.01)$. For both groups, the etiology of most cases was severe mitral regurgitation (grade 4+). Patients in the TM-VIV group were more likely to have severe tricuspid regurgitation compared to those in the SMVR group (33.3\% vs. 52\%, respectively; $\mathrm{P}=0.11$ ).

\section{Procedural and in-hospital outcomes}

Procedural details and in-hospital outcomes are summarized in Table 2. Concomitant tricuspid valve surgery was performed in $46.3 \%$ of patients in the SMVR group and 
Table 1 Baseline characteristics

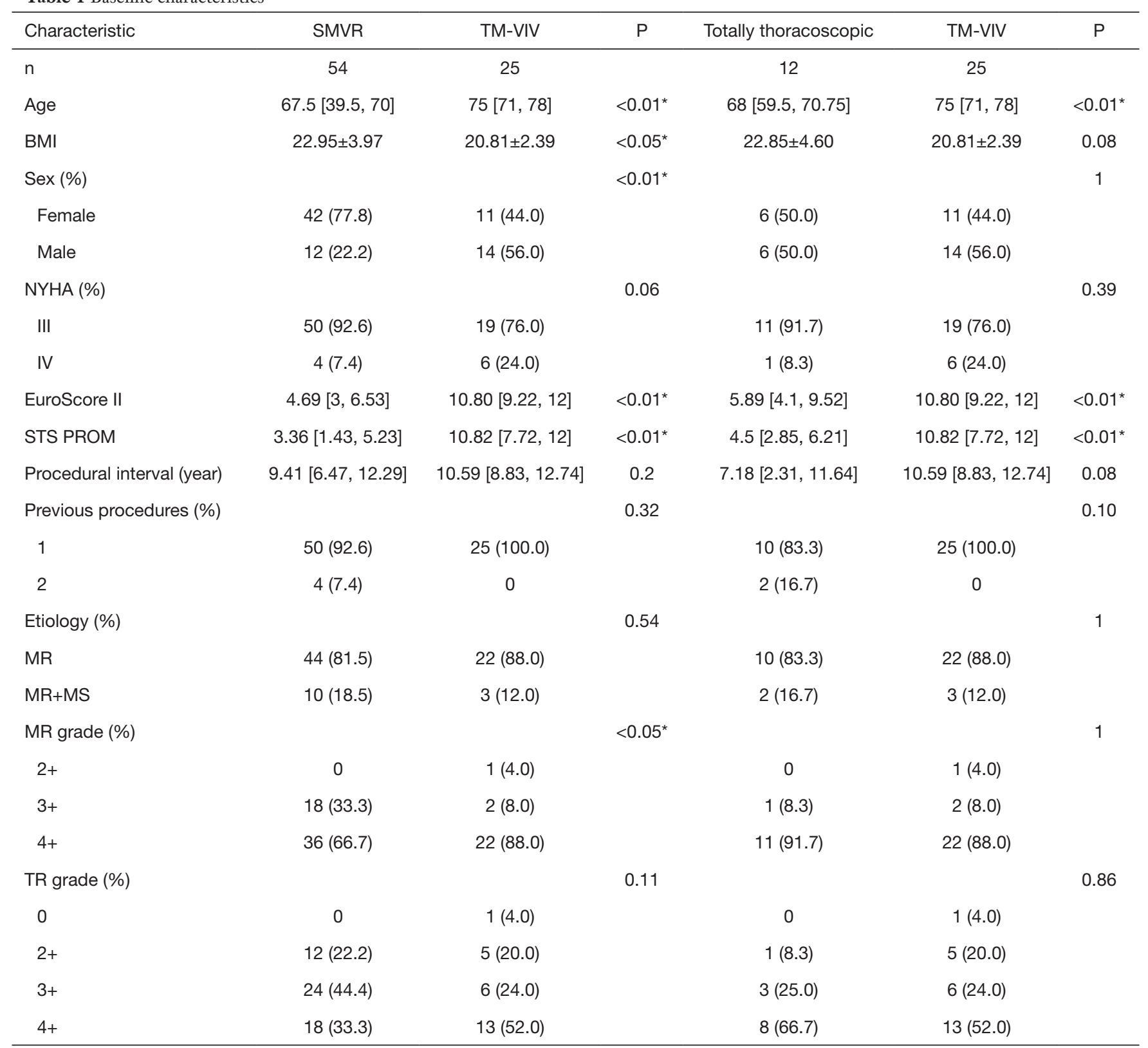

*, P<0.05. BMI, body mass index; EuroScore II, European System for Cardiac Operative Risk Evaluation II; MR, mitral regurgitation; MS, mitral stenosis; NYHA, New York Heart Association; STS PROM, The Society of Thoracic Surgeons Predicted Risk of Mortality; TR, tricuspid regurgitation; SMVR, surgical mitral valve replacement; TM-VIV, transcatheter mitral valve-in-valve implantation.

$0 \%$ of those in the TM-VIV group. In the SMVR group, myocardial protection was achieved with aortic clamping and antegrade cardioplegia in 44 patients $(81.5 \%)$. The remaining 10 patients had their procedures performed on a beating heart. About half of the patients in the SMVR group chose a mechanical prosthetic valve. The most common size of prosthetic valve was 27 (57.4\%) in SMVR group and 25
(64\%) in TM-VIV group. Table 2 shows that, in the TMVIV group, sizes 27 and 29 were used in only 2 patients (8\%) and 0 patients, respectively. The procedural success rate was $100 \%$ in the TM-VIV group, with significantly shorter procedural duration and no emergent conversion to median sternotomy.

The in-hospital mortality for the SMVR group and TM- 
Table 2 Procedure and in-hospital outcomes

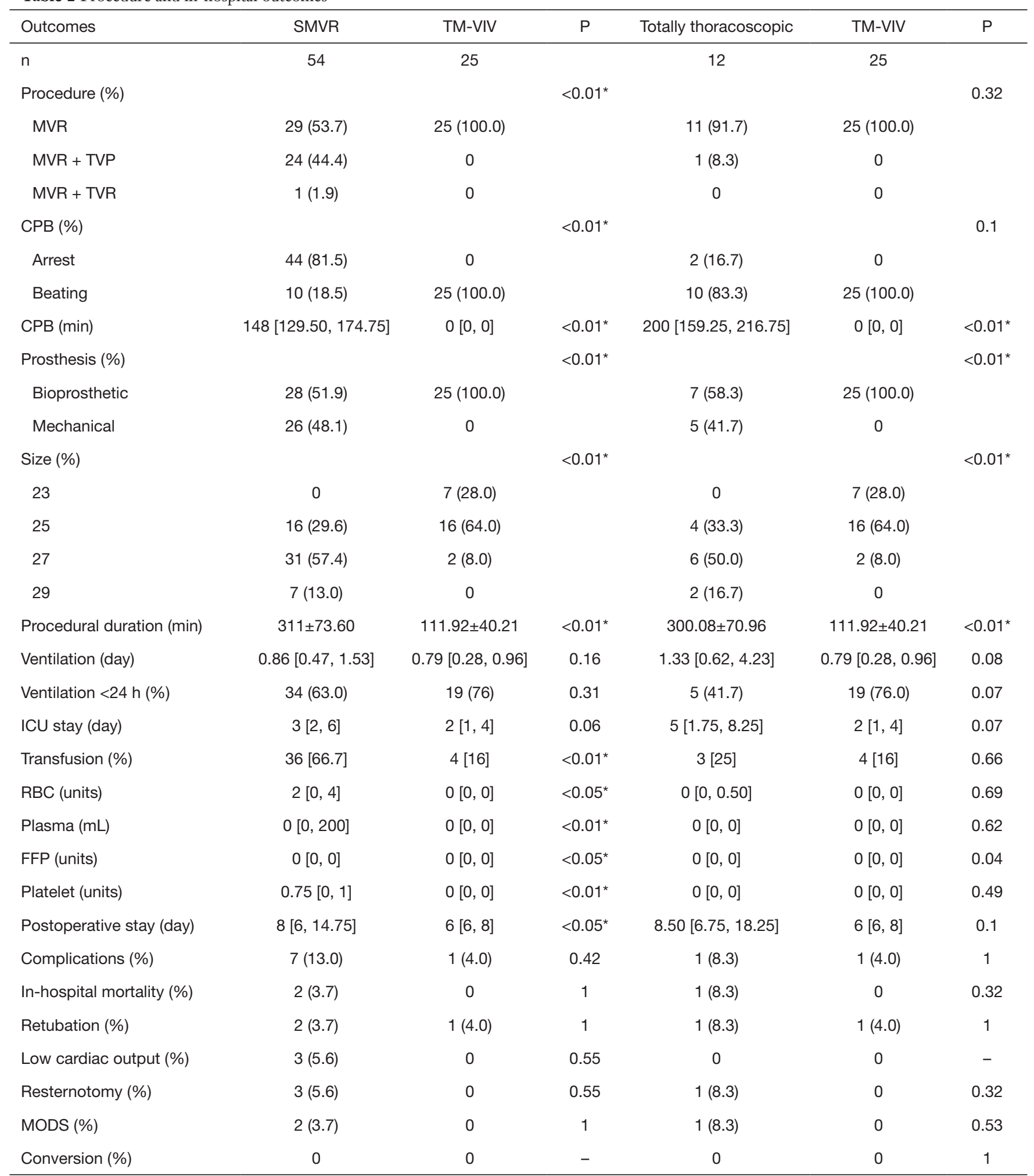

*, P<0.05. CPB, cardiopulmonary bypass; FFP, fresh frozen plasma; ICU, intensive care unit; MODS, multiple organ dysfunction syndrome; RBC, red blood cell; SMVR, surgical mitral valve replacement; TM-VIV, transcatheter mitral valve-in-valve implantation. 
Table 3 Hemodynamic data

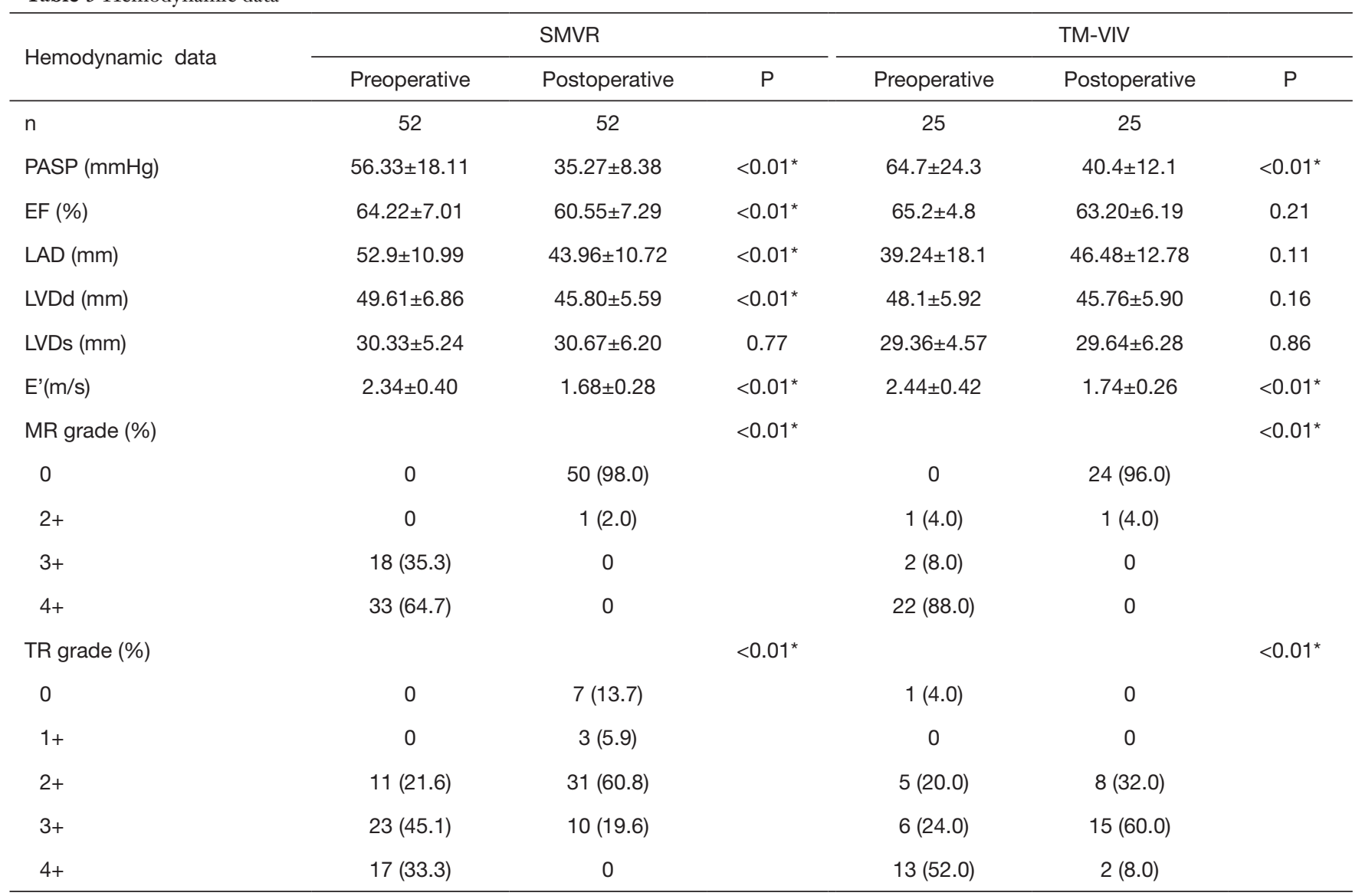

*, $\mathrm{P}<0.05$. E', mitral valve E-wave peak velocity; EF, ejection fraction; LAD, left atrial dimension; LVDd, left ventricular end-diastolic dimension; LVD, left ventricular end-systolic dimension; MR, mitral regurgitation; PASP, pulmonary artery systolic pressure; TR, tricuspid regurgitation; SMVR, surgical mitral valve replacement; TM-VIV, transcatheter mitral valve-in-valve implantation.

VIV group was $3.7 \%$ (2 patients) and $0 \%$, respectively. Compared with the SMVR group, patients in TM-VIV group were more likely to come off the ventilator within 24 postoperative hours and have a shorter ventilation time and intensive care unit stay. A significant difference in the need for blood transfusion between the SMVR group and TM-VIV group was observed $(66.7 \%$ vs. $16 \%$, respectively; $\mathrm{P}<0.01)$. Although no significant difference was observed, the SMVR group was associated with a lower incidence of major complications, including retubation, low cardiac output syndrome, resternotomy, and multiple organ dysfunction syndrome. However, the TM-VIV group had a significantly shorter postoperative in-hospital stay $(\mathrm{P}<0.05)$.

\section{Comparisons of bemodynamic data}

The changes of hemodynamic data were summarized in
Table 3. In the SMVR group, significant differences in pulmonary artery systolic pressure (PASP), ejection fraction (EF), left atrial dimension (LAD), left ventricular endsystolic dimension (LVDs), and mitral valve E-Wave peak velocity (E') were observed between preoperative and postoperative transthoracic echocardiogram. Furthermore, these echocardiographic parameters appeared to decrease in the TM-VIV group. An interesting finding is that postoperative EF significantly decreased in the SMVR group. At discharge, $2+$ residual mitral regurgitation was detected in 1 patient in each group, and $3+/ 4+$ tricuspid regurgitation was detected in 17 of 25 (68\%) TM-VIV patients and 10 of 52 (19.6\%) SMVR patients.

\section{Follow-up}

The median follow-up period was $32 \pm 22$ months for the 


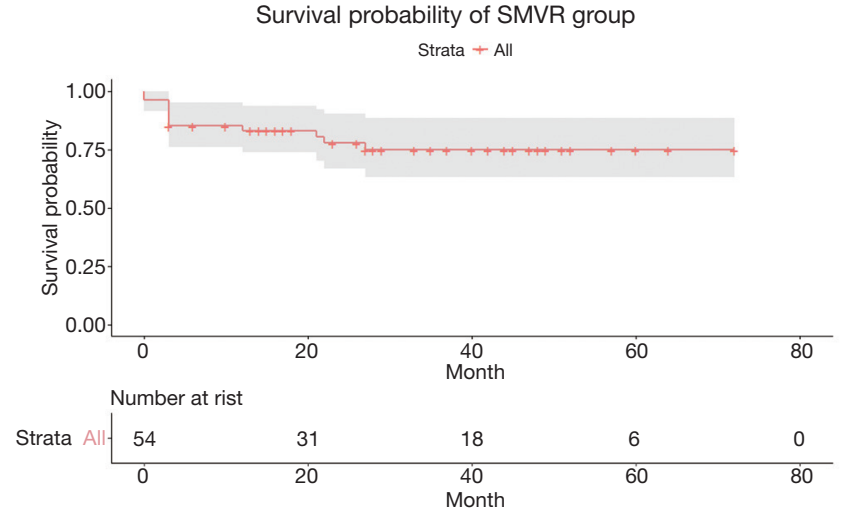

Figure 2 Kaplan-Meier plot for survival of the SMVR group. SMVR, surgical mitral valve replacement.

SMVR group and $7 \pm 3$ months for the TM-VIV group, with follow-up rates of $77 \%$ and $100 \%$, respectively; 4 patients in the SMVR group died during the follow-up period, 1 of pneumonia and 3 of endocarditis. Additionally, 5 patients developed recurrent mitral regurgitation $(2+/ 3+$ : 3 patients; 4+: 2 patients). It was related to structural deterioration and paravalvular leakage. In the TM-VIV group, residual mitral regurgitation was found in 1 patient during the followup period, and the 90 -day mortality was $0 \%$. The cause of residual mitral regurgitation may be the oversize implanted transcatheter heart valve. The Kaplan-Meier curve (Figure 2) shows that the SMVR group had a 6 -year survival of $75 \% \pm 6.4 \%$, a benchmark for the TM-VIV group in the future.

\section{Subgroup analysis}

In the SMVR group, 12 patients underwent the procedure via the totally thoracoscopic approach. Compared with the totally thoracoscopic group, the TM-VIV group was associated with higher age and higher scores on EuroScore II and STS PROM. No significant difference was detected among other patient baseline characteristics and the majority of perioperative outcomes between the groups.

\section{Discussion}

With the continual aim of minimizing trauma and improving clinical outcomes, techniques in cardiovascular surgery have been constantly improving. The concept of minimally invasive mitral valve surgery (MIMVS) was established in 1996 with the first case of mitral valvuloplasty performed through a mini-thoracotomy using videoscopy (9). Since then, MIMVS has gradually been adopted as a standard approach due to its proven feasibility and efficacy $(10,11)$. And redo mitral valve replacement is being performed in increasing numbers. The reasons for reoperation in patients with previous mitral valve replacement depend on the type of prosthetic valve. Valve thrombosis and paravalvular leak were the main reasons for reoperation in patients with prior mechanical valve, whereas structural valve deterioration was the most common reason in patients with bioprosthetic valve. However, redo mitral valve surgery is associated with increased perioperative risk, which attributes to more comorbidities, broad adhesions and technical difficulties inherited from reoperation. Although there is some research on redo mitral valve surgery via minimally invasive approaches, including right mini-thoracotomy and robotic endoscopy $(12,13)$, most surgeons are more familiar with and prefer a resternotomy approach. Since the first reported use of transapical TN-VIV procedure by Cheung (14) in 2009, TM-VIV has gained favor as a viable alternative to SMVR for high-risk and elderly patients.

Previous studies have found that the 30-day mortality for TM-VIV ranges from $0 \%$ to $10 \%(15-17)$, while a recent study with 1,576 enrolled patients who underwent TM-VIV showed a $21.7 \%$ one-year mortality (18). However, data on long-term outcomes are limited, while research directly comparing conventional and minimally invasive SMVR and TM-VIV is rare.

At our institution, application of the transcatheter approach began in 2020 and was used in $84.2 \%$ of redo mitral procedures for failed mitral bioprostheses that year. In our study, the procedure was successfully performed in every patient. The in-hospital and 90-day mortality of the TM-VIV group were both $0 \%$. One important aspect of the encouraging outcomes is that the surgically implanted radiopaque bioprosthesis can serve as a landmark for orientation and deployment under fluoroscopy. In addition, the transapical antegrade approach is much easier in terms of delivery and manipulation. However, compared with the SMVR group, there were more patients in the TM-VIV group $(68 \%)$ who experienced $3+/ 4+$ postoperative tricuspid regurgitation. This was due to concomitant transcatheter tricuspid valve surgery not being available due to a lack of commercial transcatheter tricuspid products. Further, it should be emphasized that the size of transcatheter heart valve is limited by the previous surgically implanted bioprostheses. Only 2 of 25 patients used size 27 while the others used a smaller size. In the SMVR group, it was 
possible to select the optimal size for allowing maximum blood flow. Compared to the totally thoracoscopic group, patients in the TM-VIV group were older with higher predicted mortality and similar perioperative outcomes. This suggests that TM-VIV is an alternative to minimally invasive approaches for inoperable and extremely high-risk patients. While the long-term outcomes of TM-VIV are unavailable, the 6-year survival of $75 \% \pm 6.4 \%$ in the SMVR group can serve as a benchmark in the future.

\section{Limitations}

As a retrospective research, this study inherited several limitations including selections bias, misclassification bias and recall bias. So, we took measures to solve the problem including carefully defined selection criteria, used homogeneous definitions of disease, selected subjects with equal tendency to remember and collected all data in a similar way. Another main limitation of this observational analysis is the small number of patients, with only 54, 12, and 25 patients undergoing SMVR, totally thoracoscopic SMVR, and transapical TM-VIV, respectively. Furthermore, as all transapical TM-VIV procedures were performed after January 2020, data on the long-term outcomes of transapical TM-VIV were unavailable.

\section{Conclusions}

Yearly trend analysis showed a steady increase in the volume of redo mitral procedures for mitral bioprostheses failure, with an increased proportional use of the totally thoracoscopic approach. The use of transapical TM-VIV has played a dominant role since 2020. This approach has also proven to be feasible and valid alternative to SMVR, with reduced need for blood transfusion, ventilation time, ICU stay, and postoperative in-hospital stay. As reoperation for failed bioprosthetic valve is inevitable, we recommend TM-VIV as the preferred treatment for high-risk elderly patients.

\section{Acknowledgments}

Funding: Science and Technology Program of Guangzhou (202002030039).

\section{Footnote}

Reporting Checklist: The authors have completed the
STROBE reporting checklist. Available at https://dx.doi. org/10.21037/atm-21-3118

Data Sharing Statement: Available at https://dx.doi. org/10.21037/atm-21-3118

Conflicts of Interest: All authors have completed the ICMJE uniform disclosure form (available at https://dx.doi. org/10.21037/atm-21-3118). The authors have no conflicts of interest to declare.

Ethical Statement: The authors are accountable for all aspects of the work in ensuring that questions related to the accuracy or integrity of any part of the work are appropriately investigated and resolved. The study was conducted in accordance with the Declaration of Helsinki (as revised in 2013). The study was approved by the Ethics Committee of Guangdong Provincial People's Hospital (No. KY-Q-2021-088-01) and individual consent was waived.

Open Access Statement: This is an Open Access article distributed in accordance with the Creative Commons Attribution-NonCommercial-NoDerivs 4.0 International License (CC BY-NC-ND 4.0), which permits the noncommercial replication and distribution of the article with the strict proviso that no changes or edits are made and the original work is properly cited (including links to both the formal publication through the relevant DOI and the license). See: https://creativecommons.org/licenses/by-nc-nd/4.0/.

\section{References}

1. Gummert JF, Funkat A, Krian A, et al. Cardiac surgery in Germany during 2004: a report on behalf of the German Society for Thoracic and Cardiovascular Surgery. Thorac Cardiovasc Surg 2005;53:391-9.

2. Beckmann A, Meyer R, Lewandowski J, et al. German Heart Surgery Report 2019: The Annual Updated Registry of the German Society for Thoracic and Cardiovascular Surgery. Thorac Cardiovasc Surg 2020;68:263-76.

3. Beckmann A, Meyer R, Lewandowski J, et al. German Heart Surgery Report 2017: The Annual Updated Registry of the German Society for Thoracic and Cardiovascular Surgery. Thorac Cardiovasc Surg 2018;66:608-21.

4. Funkat AK, Beckmann A, Lewandowski J, et al. Cardiac surgery in Germany during 2011: a report on behalf of the German Society for Thoracic and Cardiovascular Surgery. Thorac Cardiovasc Surg 2012;60:371-82. 
5. Brown JM, O'Brien SM, Wu C, et al. Isolated aortic valve replacement in North America comprising 108,687 patients in 10 years: changes in risks, valve types, and outcomes in the Society of Thoracic Surgeons National Database. J Thorac Cardiovasc Surg 2009;137:82-90.

6. Ghoreishi M, Dawood M, Hobbs G, et al. Repeat sternotomy: no longer a risk factor in mitral valve surgical procedures. Ann Thorac Surg 2013;96:1358-65.

7. Liu J, Wei P, Ma J, et al. Propensity-matched analysis of two port approach versus three port approach for totally thoracoscopic mitral valve replacement. J Thorac Dis 2020;12:5986-95.

8. Bapat V. Valve-in-valve apps: Why and how they were developed and how to use them. EuroIntervention 2014;10 Suppl U:U44-51.

9. Carpentier A, Loulmet D, Carpentier A, et al. [Open heart operation under videosurgery and minithoracotomy. First case (mitral valvuloplasty) operated with success]. C R Acad Sci III 1996;319:219-23.

10. Atluri P, Stetson RL, Hung G, et al. Minimally invasive mitral valve surgery is associated with equivalent cost and shorter hospital stay when compared with traditional sternotomy. J Thorac Cardiovasc Surg 2016;151:385-8.

11. Grant SW, Hickey GL, Modi P, et al. Propensity-matched analysis of minimally invasive approach versus sternotomy for mitral valve surgery. Heart 2019;105:783-9.

Cite this article as: Liu J, Wei P, Liu Y, Ma J, Wu H, Tan T, Chen Z, Chen J, Huang H, Guo H. Trends in redo mitral procedure for treating mitral bioprostheses failure: a single center's experience. Ann Transl Med 2021;9(16):1306. doi: $10.21037 /$ atm-21-3118
12. Patel NC, Hemli JM, Seetharam K, et al. Reoperative mitral valve surgery via sternotomy or right thoracotomy: A propensity-matched analysis. J Card Surg 2019;34:976-82.

13. Murphy DA, Moss E, Miller J, et al. Repeat Robotic Endoscopic Mitral Valve Operation: A Safe and Effective Strategy. Ann Thorac Surg 2018;105:1704-9.

14. Cheung A, Webb JG, Wong DR, et al. Transapical transcatheter mitral valve-in-valve implantation in a human. Ann Thorac Surg 2009;87:e18-20.

15. Wilbring M, Alexiou K, Tugtekin SM, et al. Pushing the limits-further evolutions of transcatheter valve procedures in the mitral position, including valve-in-valve, valve-inring, and valve-in-native-ring. J Thorac Cardiovasc Surg 2014;147:210-9.

16. Yoon SH, Whisenant BK, Bleiziffer S, et al. Outcomes of transcatheter mitral valve replacement for degenerated bioprostheses, failed annuloplasty rings, and mitral annular calcification. Eur Heart J 2019;40:441-51.

17. Cheung A, Webb JG, Barbanti M, et al. 5-year experience with transcatheter transapical mitral valve-in-valve implantation for bioprosthetic valve dysfunction. J Am Coll Cardiol 2013;61:1759-66.

18. Whisenant B, Kapadia SR, Eleid MF, et al. One-Year Outcomes of Mitral Valve-in-Valve Using the SAPIEN 3 Transcatheter Heart Valve. JAMA Cardiol 2020;5:1245-52. 